\title{
From paraconsistent three-valued logics to multiple-source epistemic logic
}

\author{
Davide Ciucci ${ }^{1}$ Didier Dubois ${ }^{2}$ \\ ${ }^{1}$ DISCo - Università di Milano - Bicocca, Viale Sarca 336 - U14, 20126 Milano (Italia) \\ ${ }^{2}$ IRIT, Université Paul Sabatier, 118 route de Narbonne, 31062 Toulouse cedex 9 (France)
}

\begin{abstract}
Several interpretations can be given to the third truth value in three-valued logics. Here, we consider the case when it refers to the epistemic notion of contradictory, or both true and false at the same time. We study several paraconsistent threevalued logics that carry this concern and show that they can be translated into a fragment of a simple epistemic logic where modalities can only appear in front of literals. This logic is unusual in the sense that necessity modalities distribute over disjunctions instead of conjunctions. An equivalent translation into a fragment of KD modal logic can be obtained by exchanging the role of possibility and necessity modalities, highlighting the perfect symmetry between three-valued logics of contradiction and three-valued logics of incomplete information.
\end{abstract}

\section{Introduction}

The program of paraconsistent logics after Jaśkowski [14] is to find a logic which can manage contradictions and satisfies three requirements:

\section{1. when applied to the contradictory sys- tems would not always entail their over-completeness ${ }^{1}$; \\ 2. would be rich enough to enable prac- tical inference; \\ 3. would have an intuitive justification.}

Nowadays, a consensual definition of what a paraconsistent logic is does not seem to exist, but a necessary condition is that the logical consequence relation is not explosive, that is, there can exist contradictions in the logic without implying that everything is true (point 1 above) - see introduction of [20]. Several proposals to define such a logic have been studied in literature, following different lines: discursive logic, preservationism, adaptive logics, relevant logics, many valued logics [20]. Here, we are interested in the case of many valued logics, most of which are based on a three-valued calculus. In three-valued calculi, the intuition (point three above) which is attached to the third value is not always discussed and when it is, it often assumes an epistemic flavor, which can vary from unknown, contradictory to borderline [10].

\footnotetext{
${ }^{1} \mathrm{~A}$ system is over-complete if any formula is a theorem.
}

Jaśkowski himself tried to use a third value to express paraconsistency, but he seems not to have a clear definition of what a contradiction stands for. He refers to theories with conflicting hypotheses, each one able to only partially explain the result of some experiment, or to facts that are not predictable a-priori.

D'Ottaviano and Da Costa [12] cite, as a justification for their three-valued logic $J_{3}$, the existence of contradictory theories in empirical disciplines for which we are (at the moment) not able to say which theory is the correct one, which sounds more like the idea of incomplete knowledge. In [2], the third value represents antinomies, that is, propositions that are at the same time true and false. The connectives used are Kleene ones and the same logic has been studied by Priest [17]. Although the meaning is not expressed clearly, it seems that the third value stands for both true and false, as in Belnap set-ups [6].

In the following, we try to provide a unified view of three-valued logics of paraconsistency, adopting this last interpretation of the third value: it represents the case of two sources (or two groups of sources) of information which assign a different (Boolean) truth value to a proposition (this is akin to the society semantics of Carnielli and LimaMarques [16]). We do it in the same style as we recently did for three-valued logics of incomplete information [10], namely by translating many known three-valued paraconsistent formalisms into a very simple fragment of modal logic. This translation highlights the epistemic meaning of sentences in paraconsistent logics and facilitates a comparison among them.

A simple epistemic logic MELCC ${ }^{2}$ is introduced to deal with the interpretation of the third truth value in terms of conflict between sources. It is a two-layered logic: an objective level topped by an epistemic one, with no nested modal operators. In particular, it is different from the single-layer S5 discursive logic introduced by Jaśkowksi [14]. The paper lays bare the symmetry existing between threevalued paraconsistent logics and three-valued logics of incomplete information. It is shown that indeed MELCC possesses the properties of a $\mathrm{KD}$ modal logic provided that we exchange the possibility and

\footnotetext{
${ }^{2}$ It stands for Minimal Epistemic Logic with Completeness and Conflict
} 
necessity modalities.

\section{Conflicting agents, three-valued logics and modal logic}

Let us consider a set of propositional variables $\mathcal{V}=\{a, b, c, \ldots, p, \ldots\}$ and a standard propositional language $\mathcal{L}$ built on these symbols with the Boolean connectives of conjunction $(\wedge)$, disjunction $(\vee)$, negation $(')$, and implication $(\rightarrow)$, plus tautology symbol ( $\top$ ). Suppose $n$ logically sophisticated agents (i.e. they can use classical logic) sharing the same propositional language $\mathcal{L}$, and capable to decide whether any proposition $\alpha \in \mathcal{L}$ is true or false. In other words each agent possesses complete Boolean knowledge. Let $\Omega$ be the set of interpretations of $\mathcal{L}:\{\omega: \mathcal{V} \rightarrow\{0,1\}\}$. It comes down to assuming that agent $\mathcal{A}_{i}$ believes that the real world is $w_{i} \in \Omega$. For each formula $\alpha \in \mathcal{L}$, agent $i$ can say whether it is true $\left(v_{i}(\alpha)=T_{i}\right)$ or false $\left(v_{i}(\alpha)=F_{i}\right)$. Let us consider a modal expression of the assertions made by the two agents. Let $\square_{i} \alpha$ stand for the agent $\mathcal{A}_{i}$ asserting the truth of $\alpha$. Due to the complete knowledge assumption, each $\square_{i}$ is a trivial modality, i.e. $\square_{i} \alpha \vee \square_{i} \alpha^{\prime}$ is true, so $\square_{i}$ and $\diamond_{i}$ coincide, since $\nabla_{i} \alpha:=\left(\square_{i}\left(\alpha^{\prime}\right)\right)^{\prime}=\square_{i} \alpha$. In fact each modal system coincides with classical logic.

Now, let us consider a three-valued logic $L_{3}$. We denote by $v(a)$ the truth value of the variable $a$, $v(a) \in\left\{\mathbf{0}, \mathbf{1}, \frac{\mathbf{1}}{\mathbf{2}}\right\}$. We consider that these three values can be interpreted in the light of the joint assertions of both agent:

- $v(a)=\mathbf{1}$ means that all agents say that $a$ is true $\left(v_{i}(a)=T_{i}, \forall i=1, \ldots, n\right)$;

- $v(a)=\mathbf{0}$ means that all agents say that $a$ is false $\left(v_{i}(a)=F_{i}, \forall i=1, \ldots, n\right)$;

- $v(a)=\frac{1}{2}$ means that some agents say $a$ is true, the other ones say $a$ is false.

This kind of setting was first introduced by Belnap [6] with agents having incomplete knowledge. It leads to a 4-valued truth-functional logic where the 4 values include unknown and contradictory. It also corresponds to the so-called society semantics [16] where the authors (see also Dubois [13]) indicate that only two agents are needed to render this meaning of truth-values without any loss of generality. This is what is assumed in the following. Thus, $v(a)=\mathbf{1}$ stands for the set $\left\{T_{1}, T_{2}\right\}, v(a)=\mathbf{0}$ stands for the set $\left\{F_{1}, F_{2}\right\}$, and the value of contradiction $\frac{1}{2}$ stands for either set of truth values $\left\{T_{1}, F_{2}\right\}$ or $\left\{T_{2}, F_{1}\right\}^{3}$. This is pictured in Table 1 .

Now, let us define the collective modal symbols:

- $\square p$ stands for "at least one source asserts $p$ ": $\square p:=\square_{1} p \vee \square_{2} p$.

- If, as usual, we define $\nabla p:=\left(\square\left(p^{\prime}\right)\right)^{\prime}$, we have that $\diamond p=\diamond_{1} p \wedge \diamond_{2} p=\square_{1} p \wedge \square_{2} p$ here (since

\footnotetext{
${ }^{3}$ This convention is in accordance with Belnap logic where $\frac{\mathbf{1}}{\mathbf{2}}$ is named BOTH and contrary to the approach in [13] where contradiction is represented by the emptyset.
}

\begin{tabular}{c|c|c} 
& $T_{2}$ & $F_{2}$ \\
\hline$T_{1}$ & $\mathbf{1}$ & $\frac{1}{\mathbf{2}}$ \\
\hline$F_{1}$ & $\frac{1}{\mathbf{2}}$ & $\mathbf{0}$
\end{tabular}

Table 1: Epistemic values from two complete sources

each agent has complete knowledge), that is "both sources assert $p "$.

We can then encode the assignment of $L_{3}$ truthvalues to a (Boolean) propositional variable $a$ by means of the global modalities $\square$ and $\diamond$. To this end, on top of the standard propositional language $\mathcal{L}$, we build another propositional language $\mathcal{L}_{\square}$ which encapsulates $\mathcal{L}$. It is another propositional language based on a set of variables $\mathcal{V}_{\square}=\{\square \alpha$ : $\alpha \in \mathcal{L}\}$, where the modality operator $\square$ expresses necessity, as well as the classical connectives.

Let $S \subseteq\left\{\mathbf{0}, \mathbf{1}, \frac{\mathbf{1}}{\mathbf{2}}\right\}$ be a subset of $L_{3}$ truth-values. We denote by $\mathcal{T}(v(a) \in S)$ the modal translation of the set $\{v: v(a) \in S\}$ corresponding to the statement $v(a) \in S$ by an agent. Due to the proposed understanding of the three truth-values, the translation of the assignment of a subset of ternary truthvalues to an atom is a function $\mathcal{T}: 2^{\left(\left\{\mathbf{0}, \mathbf{1}, \frac{\mathbf{1}}{\mathbf{2}}\right\}^{\mathcal{V}}\right)} \rightarrow \mathcal{L}_{\square}$ from subsets of ternary valuations to the modal language $\mathcal{L}_{\square}$ such that:

$$
\begin{array}{llrl}
\mathcal{T}\left(v(a) \geq \frac{\mathbf{1}}{2}\right)=\square a & \mathcal{T}\left(v(a) \leq \frac{\mathbf{1}}{\mathbf{2}}\right) & =\square a^{\prime} \\
\mathcal{T}(v(a) & =\mathbf{1})=\diamond a & \mathcal{T}(v(a)=\mathbf{0})=\diamond a^{\prime} \\
\mathcal{T}\left(v(a)=\frac{\mathbf{1}}{\mathbf{2}}\right)=\square a \wedge \square a^{\prime} & \mathcal{T}(v(a) \in \emptyset)=\diamond \perp \\
\mathcal{T}(v(a) \in\{\mathbf{0}, \mathbf{1}\})=\diamond a \vee \diamond b & \mathcal{T}(v(a) \geq \mathbf{0})=\square \top
\end{array}
$$

So, $\frac{1}{2}$ means that one source asserts $a$ and the other $a^{\prime}$. Moreover, since in paraconsistent logics designated values are usually $\frac{\mathbf{1}}{\mathbf{2}}$ and $\mathbf{1}$, we see that $\square a$ corresponds to asserting $a$ in a paraconsistent logic. Note that this is NOT a syntactic translation from one logic to another: it provides a tool for expressing the semantics of one logic into the syntax of another one.

\section{The target modal system}

We denote by $\alpha, \beta, \ldots$ the propositional formulae of $\mathcal{L}$, and $\phi, \psi, \ldots$ the modal formulae of $\mathcal{V}_{\square}$. A logic that uses the language $\mathcal{L}_{\square}$ defined above, called MELC [13] has been proposed as a minimal epistemic logic with conflicts. It uses the propositional logic axioms, and some modal ones:

$$
\begin{gathered}
\text { 1. } \phi \rightarrow(\psi \rightarrow \phi) \\
\text { 2. }(\psi \rightarrow(\phi \rightarrow \mu)) \rightarrow((\psi \rightarrow \phi) \rightarrow(\psi \rightarrow \mu)) \\
\text { 3. }\left(\phi^{\prime} \rightarrow \psi^{\prime}\right) \rightarrow(\psi \rightarrow \phi) \\
(\mathrm{RE}) \square \alpha \equiv \square \beta \text { if and only if } \vdash \alpha \equiv \beta \\
(\mathrm{RM}) \square \alpha \rightarrow \square \beta \text { if } \vdash \alpha \rightarrow \beta \text { in PL }
\end{gathered}
$$


(3-C) $\square \alpha \wedge \square \beta \wedge \square \gamma \rightarrow \square(\alpha \wedge \beta) \vee \square(\alpha \wedge \gamma) \vee \square(\beta \wedge \gamma)$

(N) $\square \top$;

$(\mathrm{POS}) \diamond \top$

The only rule is modus ponens: If $\psi$ and $\psi \rightarrow \phi$ then $\phi$. This is a fragment of the non-regular logic EMN [8] (except for axiom 3-C). Semantics are usually expressed in terms of neighborhood semantics, but they can be related to the multisource environment suggested above. Concerning semantics, denote the set of classical models of $\alpha$ by $[\alpha]=\{\omega: \omega \models \alpha\}$. Each source has its own epistemic state $E_{i} \subseteq \Omega$. A (meta)-interpretation of $\mathcal{L}_{\square}$ is a pair $\left(E_{1}, E_{2}\right)$, with $E_{1}, E_{2} \neq \emptyset$. We define satisfiability as:

- $\left(E_{1}, E_{2}\right) \models \square \alpha$ if $E_{1} \subseteq[\alpha]$ or $E_{2} \subseteq[\alpha]$;

- $\left(E_{1}, E_{2}\right) \models \phi \wedge \psi$ if $\left(E_{1}, E_{2}\right) \models \phi$ and $\left(E_{1}, E_{2}\right) \models \psi$;

- $\left(E_{1}, E_{2}\right) \models \phi^{\prime}$ if $\left(E_{1}, E_{2}\right) \models \phi$ is false.

In this setting, axiom (3-C) expresses the fact that there are exactly two sources. $\square \alpha$ can be expressed as the disjunction $\square_{1} \alpha \vee \square_{2} \alpha$ corresponding to KD modalities [13]. Semantic inference of a formula from a modal base $B$ is defined in the usual way: $B \models \phi$ if and only if $\forall E_{1}, E_{2} \neq \emptyset \subseteq \Omega$, if $\left(E_{1}, E_{2}\right) \models \psi, \forall \psi \in B$, then $\left(E_{1}, E_{2}\right) \models \phi$.

The logic we want to consider here is an extension of MELC that takes into account the fact that we can have contradictions and that the knowledge of each agent is complete. As a consequence, the modalities constructed in the previous section have distinguished properties. Due to the fact that both sources are complete, there is the following (unusual) property, for general Boolean propositions.

Proposition 3.1. For any proposition, $\square \alpha \vee \square \beta$ is equivalent to $\square(\alpha \vee \beta)$.

Proof. This is easily derived from the definition of $\square$ and the fact that we suppose the knowledge complete, that is for each agent $\mathcal{A}_{i}$ it holds for $\square_{i}$.

However, we do not have that $\square \alpha \wedge \square \beta$ is equivalent to $\square(\alpha \wedge \beta)$. Likewise, the following property holds : $\square \alpha \vee \square \alpha^{\prime}$ is a tautology. From it, we can also derive $\diamond \alpha \rightarrow \square \alpha$, which represents the idea that $\diamond$ stands for an agreement of the two sources, both asserting that $\alpha$ is true, whereas $\square$ just expresses the idea that one of the two is true, but not necessarily both. Finally the MELCC system is MELC plus deviant modal axioms:

$$
\begin{aligned}
& (N \vee) \vdash \square(\alpha \vee \beta) \rightarrow(\square \alpha \vee \square \beta) ; \\
& \left(D^{t}\right) \vdash \diamond \alpha \rightarrow \square \alpha .
\end{aligned}
$$

The semantics of this system is the same as MELC, except that now, $\left(E_{1}, E_{2}\right)=\left(\left\{w_{1}\right\},\left\{w_{2}\right\}\right)$ in the above definitions of satisfiability and semantic inference. This is enforced by axiom $(N \vee)$. Moreover, $(N)$ becomes a redundant axiom. At this point, we are equipped with sufficient tools to make sense of three-valued paraconsistent logics inside the multisource epistemic logic MELCC, via a translation of their connectives and axioms. We start with the simplest one, that is Priest logic.

\section{Translation of Priest logic}

Priest [17] attaches to the third truth value the meaning of a paradox; it refers to sentences that are "both true and false" His intention is to "isolate paradoxes and prevent them from contaminating everything else".

To define the basic connectives, his reasoning implicitly assumes that a sentence can have the following values $\{\mathbf{0}\},\{\mathbf{0}, \mathbf{1}\},\{\mathbf{1}\}$ and then he extends the classical Boolean truth tables to set-valued arguments (see also $[15,13]$ ). So if $A$ is true and $B$ paradoxical, he gets $\{\mathbf{1}\} \wedge\{\mathbf{0}, \mathbf{1}\}=\{\mathbf{1} \wedge \mathbf{0}, \mathbf{1} \wedge \mathbf{1}\}=\{\mathbf{0}, \mathbf{1}\}$, i.e. paradoxical. Hence, Kleene strong truth tables are recovered, that is the minimum $\sqcap$, the maximum $\sqcup$, and the involutive negation $\neg$, the material implication $a \rightarrow_{P} b:=\neg a \sqcup b$ of a Kleene lattice. However, in his system both $\mathbf{1}$ and $\frac{\mathbf{1}}{\mathbf{2}}$ are designated. In fact, the notion of semantic inference is defined as follows:

Definition 1. If $B$ is a set of propositions in Kleene logic, then $B \vDash_{P} \alpha$ if and only if there does not exist an interpretation $v$ such that $v(\alpha)=\mathbf{0}$ and for all $\beta \in B, v(\beta) \in\left\{\mathbf{1}, \frac{\mathbf{1}}{\mathbf{2}}\right\}$.

In other words, if $v(\beta) \geq \frac{\mathbf{1}}{\mathbf{2}}, \forall \beta \in B$ then $v(\alpha) \geq$ $\frac{\mathbf{1}}{\mathbf{2}}$. As a result, all Boolean tautologies are still valid, but modus ponens does not hold any longer. A similar intuition of the third value is given by Asenjo [2] to deal with antinomies and he also comes up with Kleene truth tables.

If the knowledge base $B$ in Priest logic contains an atom $a$, it means that $v(a) \geq \frac{\mathbf{1}}{\mathbf{2}}$, that is, we write $\square a$ in MELCC. If $B$ contains the conjunction of two atoms $a \sqcap b$, this is translated as:

$$
\mathcal{T}\left(v(a \sqcap b) \geq \frac{\mathbf{1}}{\mathbf{2}}\right)=\square a \wedge \square b
$$

and similarly for the disjunction. Note that $\mathcal{T}(v(a \sqcap$ $b)=\mathbf{1})=\diamond a \wedge \diamond b$. Translations of formulae declared as true in Priest Logic are defined recursively.

$$
\begin{aligned}
\mathcal{T}(v(\alpha \sqcap \beta) \geq i) & =\mathcal{T}(v(\alpha) \geq i) \wedge \mathcal{T}(v(\beta) \geq i), i \geq \frac{\mathbf{1}}{\mathbf{2}} \\
\mathcal{T}(v(\alpha \sqcup \beta) \geq i) & =\mathcal{T}(v(\alpha) \geq i) \vee \mathcal{T}(v(\beta) \geq i), i \geq \frac{\mathbf{1}}{\mathbf{2}} \\
\mathcal{T}\left(v(\neg \alpha) \geq \frac{\mathbf{1}}{\mathbf{2}}\right) & =\mathcal{T}\left(v(\alpha) \leq \frac{\mathbf{1}}{\mathbf{2}}\right)
\end{aligned}
$$

Note that $\mathcal{T}\left(v(\neg \alpha) \geq \frac{\mathbf{1}}{\mathbf{2}}\right)=(\mathcal{T}(v(\alpha)=\mathbf{1}))^{\prime}$. Implication statements can be translated by means of other connectives or directly:

$\mathcal{T}\left(v\left(\alpha \rightarrow_{P} \beta\right) \geq \frac{\mathbf{1}}{\mathbf{2}}\right)=\mathcal{T}(v(\alpha)=\mathbf{1}) \rightarrow \mathcal{T}\left(v(\beta) \geq \frac{\mathbf{1}}{\mathbf{2}}\right)$.

Applied to atoms $a$ and $b$, implication statement $a \rightarrow_{P} b \in B$ translates into:

$$
\mathcal{T}\left(v\left(a \rightarrow_{P} b\right) \geq \frac{\mathbf{1}}{\mathbf{2}}\right)=\diamond a \rightarrow \square b=\square a^{\prime} \vee \square b
$$


Note that in contrast $\mathcal{T}\left(v\left(\begin{array}{lll}\alpha \rightarrow_{P} & \beta\end{array}\right)=\mathbf{1}\right)=$ $\mathcal{T}\left(v(\alpha) \geq \frac{\mathbf{1}}{\mathbf{2}}\right) \rightarrow \mathcal{T}(v(\beta)=\mathbf{1})$, so that $\mathcal{T}\left(v\left(a \rightarrow_{P}\right.\right.$ $b)=\mathbf{1})=\square a \rightarrow \diamond b=\diamond a^{\prime} \vee \diamond b$. Note that all subsets of $\left(\left\{\mathbf{0}, \mathbf{1}, \frac{\mathbf{1}}{\mathbf{2}}\right\}^{\mathcal{V}}\right)$ can be expressed as $\{v: v(\alpha \in$ $S)\}$ for some subset $S$ of truth-values and some Priest logic formula $\alpha$. So, by extending function $\mathcal{T}$ to all formulas built with connectives of Priest logic, we make it a mapping.

Let $\alpha$ be a formula in conjunctive normal form (without simplifying the terms of the form $a \sqcap \neg a$ ). Now, since designated truth values are $\mathbf{1}$ and $\frac{\mathbf{1}}{\mathbf{2}}$, its translation into MELCC consists in the same classical conjunction of disjunctions where we put a modality $\square$ in front of all literals. So, if we replace all literals $l$ by $\square l$, any propositional tautology in this form remains a tautology in MELCC. Clearly, the fragment of MELCC we can capture is just given by the conjunction and disjunction of literals preceded by a $\square$. That is: $\square a\left|\square a^{\prime}\right| \psi \vee \phi \mid \psi \wedge \phi$. In Priest logic, we do not have modus ponens. This is captured in MELCC, noticing that from $\square a$ and $\diamond a \rightarrow \square b$ it does not follow $\square b$. Similarly, $\alpha \sqcap \neg \alpha \vdash \beta$ does not hold, and this is expressed by the fact that $\square a \wedge \square a^{\prime}$ is not a contradiction in MELCC.

Given a formula $\alpha$ in Priest logic and a model for its translation into MELCC, we are now able to define a three-valued interpretation for $\alpha$.

Proposition 4.1. Let $\alpha$ be a formula in Priest logic. For any model $\left(w_{1}, w_{2}\right)$ of $\mathcal{T}\left(v(\alpha) \geq \frac{\mathbf{1}}{\mathbf{2}}\right)$ the interpretation $v_{\left(w_{1}, w_{2}\right)}$ defined below is a model of $\alpha: \forall a$,

$$
v_{\left(w_{1}, w_{2}\right)}(a)= \begin{cases}\mathbf{1} & \left(w_{1}, w_{2}\right) \vDash \diamond a \\ & \left(\text { that } i s, w_{1} \models a \text { and } w_{2} \models a\right) \\ \mathbf{0} \quad\left(w_{1}, w_{2}\right) \models \diamond \neg a \\ \quad\left(\text { that } i s, w_{1} \models a \text { and } w_{2} \models a\right) \\ \frac{1}{\mathbf{1}} \quad \text { otherwise }\end{cases}
$$

The other way around does not hold, strictly speaking. Indeed, given a pair $\left(w_{1}, w_{2}\right)$ of Boolean evaluations, we are able to define the corresponding three-valued valuations. On the contrary, if we have a three-valued valuation, we are not able to distinguish between the two sources that generated it. However, this is not a real issue : in MELCC, the two models $\left(w_{1}, w_{2}\right)$ and $\left(w_{2}, w_{1}\right)$ satisfy the same set of formulas and are indeed indistinguishable; in fact MELCC models are to be viewed, like two-element models of MEL, as doubletons of interpretations (i.e. sets, not pairs). In fact, another translation is possible into MEL [10]. It is exactly like the above translation, exchanging $\square$ and $\diamond$. The two translations are then isomorphic.

\section{Translation of $\mathbf{A}$ and $J_{3}$ logics}

This is the logic proposed by Asenjo and Tamburino in [3]. Connectives are $\min , \max$, involutive nega- tion and Jaskowksi implication ${ }^{4}$ : the designated

\begin{tabular}{c|c|c|c}
$\rightarrow_{J}$ & $\mathbf{0}$ & $\frac{1}{2}$ & $\mathbf{1}$ \\
\hline 0 & 1 & 1 & 1 \\
\hline$\frac{1}{2}$ & 0 & $\frac{1}{2}$ & 1 \\
\hline 1 & 0 & $\frac{1}{2}$ & 1
\end{tabular}

Table 2: Jaśkowski implication

values are $\mathbf{1}$ and $\frac{\mathbf{1}}{\mathbf{2}}$. The only difference with respect to Priest is the introduction of the new implication $\rightarrow_{J}$, whose translation into MELCC is as follows.

$\mathcal{T}\left(v\left(\alpha \rightarrow_{J} \beta\right)=\mathbf{1}\right)=\mathcal{T}\left(v(\alpha) \geq \frac{\mathbf{1}}{\mathbf{2}}\right) \rightarrow \mathcal{T}(v(\beta)=\mathbf{1})$

$\mathcal{T}\left(v\left(\alpha \rightarrow_{J} \beta\right) \geq \frac{\mathbf{1}}{\mathbf{2}}\right)=\mathcal{T}\left(v(\alpha) \geq \frac{\mathbf{1}}{\mathbf{2}}\right) \rightarrow \mathcal{T}\left(v(\beta) \geq \frac{\mathbf{1}}{\mathbf{2}}\right)$

If $\alpha=a, \beta=b$ are atoms, we obtain respectively $\square a \rightarrow \diamond b$ and $\square a \rightarrow \square b$. Clearly, in this logic modus ponens does hold. Indeed, it corresponds in MELCC to state that from $\mathcal{T}(v(\alpha) \geq$ $\left.\frac{\mathbf{1}}{\mathbf{2}}\right) \rightarrow \mathcal{T}(v(\beta)=\mathbf{1})$ and $\mathcal{T}\left(v(\alpha) \geq \frac{\mathbf{1}}{\mathbf{2}}\right)$, it follows $\mathcal{T}(v(\beta)=\mathbf{1})$. On the other hand, not all classical tautologies are valid. But if we consider the fragment of $\mathbf{A}$ without $\neg$ we have that all classical tautologies are still valid in $\mathbf{A}$ (this has also been stated in [12] for the equivalent logic $J_{3}$, see below).

We note that the negation $\sim \alpha:=\alpha \rightarrow_{J} \mathbf{0}$ is an intuitionistic one and we have the equality $\alpha \rightarrow_{J} \beta=\sim \alpha \vee \beta$. At the atomic level, the translation into MELCC of the negation $\sim a$ is $\mathcal{T}(v(\sim a)=\mathbf{1})=\mathcal{T}\left(v(\sim a) \geq \frac{\mathbf{1}}{\mathbf{2}}\right)=(\diamond a)^{\prime}=\square a^{\prime}$. So the fragment in MELCC which corresponds to the A logic is $\square a\left|\square a^{\prime}\right| \phi^{\prime}|\phi \wedge \psi| \phi \vee \psi$, that is the language of MELCC where modalities are in front of literals only. Proposition 4.1 can be extended to Jaśkowski implication.

In [12] the authors introduce the logic $J_{3}$ to answer points 1-3 of Jaśkowski. The primitive connectives are max, the involutive negation and the unary strengthening operator $\nabla$ such that $\nabla \mathbf{0}=\mathbf{0}$, $\nabla \frac{1}{2}=\nabla \mathbf{1}=\mathbf{1}$. Among the derived operations, it is worth to mention Jaśkowski implication as $a \rightarrow_{J} b:=\neg \nabla a \vee b$ and Eukasiewicz implication as $a \rightarrow_{L} b:=(\nabla \neg a \vee b) \wedge(\nabla b \vee \neg a)$. If we explicitly express $\nabla$ in MELCC we have that for a given atom $a, \nabla a$ is translated into $\square a$. Further, it can be easily seen that $\nabla$ is also definable in $\mathbf{A}$ as $\nabla a=\neg\left(a \rightarrow_{J} \mathbf{0}\right)$, so $\mathbf{A}$ and $J_{3}$ have the same connectives and all the results proved for $\mathbf{A}$ also hold for $J_{3}$.

Finally, in [3] an axiomatic system is proposed for the logic $\mathbf{A}$, that takes into account the distinction between antinomic vs. non-antinomic statements, distinction at the basis of the logic $\mathbf{A}$. This difference looks hardly expressible in MELCC, in particular the axiom asserting $B_{i} \sqcap \neg B_{i}$ with $B_{i}$ an atomic antinomy (that is both $B_{i}$ and $\neg B_{i}$ are provable).

\footnotetext{
${ }^{4}$ Note that Jaśkowski traces back this implication to Słupecki
} 


\section{Translation of RM3 (Sobociński) logic}

The same connectives that define $\mathbf{A}$, also define the relevance logic $\mathrm{RM}_{3} \rightarrow^{J}$ [4] $\left(\rightarrow_{J}\right.$ is denoted by $\supset$ in the original paper), which is also equivalent to RM3 $[1,7]$, that is to Sobociński $[19,4]$ logic through the following mutual definitions:

$$
\begin{aligned}
& p \rightarrow_{S} q:=\left(p \rightarrow_{J} q\right) \wedge\left(\neg q \rightarrow_{J} \neg p\right) \\
& p \rightarrow_{J} q:=q \vee\left(p \rightarrow_{S} q\right)
\end{aligned}
$$

Thus, even if Sobociński and RM3 were not conceived to deal with paraconsistency, they can play a role in this framework: in [4], it is claimed that $\mathrm{RM}_{3}^{\rightarrow}$ "might be considered an optimal paraconsistent logic".

As a consequence of all these equivalences, all above translation results also apply to RM3 and Sobociński logic. In particular, Sobociński implication whose truth table is on Table 3 can be translated as

\begin{tabular}{|c|c|c|c|c|c|c|c|c|c|}
\hline$\rightarrow_{S}$ & 0 & $\frac{1}{2}$ & \multicolumn{2}{|c|}{1} & \multicolumn{2}{|c|}{$\wedge_{S}$} & 0 & $\frac{1}{2}$ & 1 \\
\hline 0 & 1 & 1 & \multicolumn{2}{|c|}{1} & \multicolumn{2}{|c|}{0} & 0 & 0 & 0 \\
\hline$\frac{1}{2}$ & 0 & $\frac{1}{2}$ & \multicolumn{2}{|c|}{1} & & & 0 & $\frac{1}{2}$ & 1 \\
\hline \multirow[t]{5}{*}{1} & 0 & 0 & \multicolumn{2}{|c|}{1} & & & 0 & 1 & 1 \\
\hline & & & & 0 & $\frac{1}{2}$ & 1 & & & \\
\hline & & & & 0 & 0 & 1 & & & \\
\hline & & & & 0 & $\frac{1}{2}$ & 1 & & & \\
\hline & & & & 1 & 1 & $\mathbf{1}$ & & & \\
\hline
\end{tabular}

$$
\begin{aligned}
\mathcal{T}\left(v\left(\alpha \rightarrow_{S} \beta\right)=\mathbf{1}\right) & =\left[\mathcal{T}\left(v(\alpha) \geq \frac{\mathbf{1}}{\mathbf{2}}\right) \rightarrow \mathcal{T}(v(\beta)=\mathbf{1})\right] \\
\mathcal{T}\left(v\left(\alpha \rightarrow_{S} \beta\right) \geq \frac{\mathbf{1}}{\mathbf{2}}\right) & =\left[\mathcal{T}\left(v(\alpha) \geq \frac{\mathbf{1}}{\mathbf{2}}\right) \rightarrow \mathcal{T}\left(v(\beta) \geq \frac{\mathbf{1}}{\mathbf{2}}\right)\right] \\
& \wedge[\mathcal{T}(v(\alpha)=\mathbf{1}) \rightarrow \mathcal{T}(v(\beta)=\mathbf{1})]
\end{aligned}
$$

Table 3: Sobociński implication, conjunction and disjunction

For atoms, it respectively yields $\square a \rightarrow \diamond b$ and $(\square a \rightarrow \square b) \wedge(\diamond a \rightarrow \diamond b)$.

Sobociński conjunction and disjunction can be translated into MEL from Table 3 or by means of the definition $\alpha \wedge_{S} \beta=\neg\left(\alpha \rightarrow_{S} \neg \beta\right)$ and de Morgan properties.

On atoms they read as

$$
\begin{aligned}
& \mathcal{T}\left(v\left(a \wedge_{S} b\right)=\mathbf{1}\right)=(\square a \wedge \diamond b) \vee(\diamond a \wedge \square b) \\
& \mathcal{T}\left(v\left(a \wedge_{S} b\right) \geq \frac{\mathbf{1}}{\mathbf{2}}\right)=\square a \wedge \square b \\
& \mathcal{T}\left(v\left(a \vee_{S} b\right)=\mathbf{1}\right)=\diamond a \vee \diamond b \\
& \mathcal{T}\left(v\left(a \vee_{S} b\right) \geq \frac{\mathbf{1}}{\mathbf{2}}\right)=\diamond a \vee \diamond b \vee\left(\square a \wedge \square a^{\prime} \wedge \square b \wedge \square b^{\prime}\right)
\end{aligned}
$$

As far as the axiom system is concerned and due to the equivalence between the RM3 and Sobociński logics, we just consider the axiom system of RM3 [7].

$$
\begin{gathered}
\alpha \rightarrow_{S} \alpha ; \\
\left(\alpha \sqcap\left(\alpha \rightarrow_{S} \beta\right)\right) \rightarrow_{S} \beta ; \\
\alpha \sqcap \beta \rightarrow_{S} \alpha ; \\
\alpha \sqcap \beta \rightarrow_{S} \beta ; \\
\left(\left(\alpha \rightarrow_{S} \beta\right) \sqcap\left(\alpha \rightarrow_{S} \gamma\right)\right) \rightarrow_{S}\left(\alpha \rightarrow_{S}(\beta \sqcap \gamma)\right) ; \\
\left(\alpha \rightarrow_{S} \neg \alpha\right) \rightarrow_{S} \neg \alpha ; \\
\left(\alpha \rightarrow_{S} \neg \beta\right) \rightarrow\left(\beta \rightarrow_{S} \neg \alpha\right) ; \\
\left(\neg \neg \alpha \rightarrow_{S} \alpha\right) ; \\
(\neg \alpha \sqcap \beta) \rightarrow(\alpha \rightarrow \beta) ;
\end{gathered}
$$

Inference rules are:

1. $\alpha$ and $\alpha \rightarrow_{S} \beta$ implies $\beta$

2. $\alpha$ and $\beta$ implies $\alpha \sqcap \beta$

3. $\alpha \rightarrow_{S} \beta$ and $\gamma \rightarrow_{S} \delta$ implies $\left(\beta \rightarrow_{S} \gamma\right) \rightarrow_{S}$ $\left(\alpha \rightarrow_{S} \delta\right)$

Lemma 1. If $\alpha$ is a formula in RM3 logic, then $\mathcal{T}\left(v(\alpha) \geq \frac{\mathbf{1}}{\mathbf{2}}\right) \vee \mathcal{T}\left(v(\alpha) \leq \frac{\mathbf{1}}{\mathbf{2}}\right)$ is a tautology in $M E L C C$.

Proof. The proof (omitted here) is by induction on the structure of $\alpha$.

Proposition 6.1. If $A$ is an axiom in RM3 logic, then $\mathcal{T}\left(v(A) \geq \frac{\mathbf{1}}{\mathbf{2}}\right)$ is a tautology in $M E L C C$.

Proof. Axioms (R1), (R3), (R4) and (R8) are easily proved.

Axiom (R2). It is the conjunction of two tautologies. The first one follows from the fact that (R2) holds in Boolean logic and the second one by Lemma 1 .

Axiom (R5) and (R7) are the conjunctions of two tautologies that easily follows by the fact that (R5) is a Boolean theorem.

Axiom (R6) is the conjunction of two tautologies. The first one is just lemma 1 and the second one the Boolean version of axiom (R6).

Axiom (R9) is the conjunction of two tautologies. The second one is just axiom R9 in Boolean logic. The first half is:

$\left(\mathcal{T}\left(v(\neg \alpha) \geq \frac{\mathbf{1}}{\mathbf{2}}\right) \wedge \mathcal{T}\left(v(\beta) \geq \frac{\mathbf{1}}{\mathbf{2}}\right)\right) \rightarrow\left[\left(\mathcal{T}\left(v(\alpha) \geq \frac{\mathbf{1}}{\mathbf{2}}\right) \rightarrow\right.\right.$ $\left.\mathcal{T}\left(v(\beta) \geq \frac{\mathbf{1}}{\mathbf{2}}\right)\right) \wedge(\mathcal{T}(v(\alpha)=\mathbf{1}) \rightarrow \mathcal{T}(v(\beta)=\mathbf{1})]$,

that is:

$\mathcal{T}(v(\alpha)=\mathbf{1}) \vee\left(\mathcal{T}\left(v(\beta) \geq \frac{\mathbf{1}}{\mathbf{2}}\right)\right)^{\prime} \vee\left[\left(\mathcal{T}\left(v(\alpha) \geq \frac{\mathbf{1}}{\mathbf{2}}\right)^{\prime} \vee\right.\right.$

$\left.\mathcal{T}\left(v(\beta) \geq \frac{\mathbf{1}}{\mathbf{2}}\right)\right) \wedge\left(\mathcal{T}(v(\alpha)=\mathbf{1})^{\prime} \vee \mathcal{T}(v(\beta)=\mathbf{1})\right]$.

Then by distributivity, we can simplify as

$\mathcal{T}(v(\alpha)=\mathbf{1}) \vee\left(\mathcal{T}\left(v(\beta) \geq \frac{\mathbf{1}}{\mathbf{2}}\right)\right)^{\prime} \vee\left(\mathcal{T}(v(\alpha)=\mathbf{1})^{\prime} \vee\right.$ $\mathcal{T}(v(\beta)=\mathbf{1})$,

which is a Boolean tautology.

Axiom (10) is translated into the following two tautologies:

$\mathcal{T}(v(\alpha)=\mathbf{1}) \vee\left(\mathcal{T}\left(v(\alpha) \geq \frac{\mathbf{1}}{\mathbf{2}}\right) \vee\left[\left(\left(\mathcal{T}\left(v(\alpha) \geq \frac{\mathbf{1}}{\mathbf{2}}\right)^{\prime} \vee\right.\right.\right.\right.$ $\left.\mathcal{T}\left(v\left(\beta \geq \frac{\mathbf{1}}{\mathbf{2}}\right)\right) \wedge\left(\mathcal{T}(v(\alpha)=1)^{\prime} \vee \mathcal{T}(v(\beta)=\mathbf{1})\right)\right]$ and

$\mathcal{T}\left(v(\alpha) \geq \frac{\mathbf{1}}{\mathbf{2}}\right) \vee \mathcal{T}(v(\alpha)=\mathbf{1}) \vee\left(\mathcal{T}\left(v(\alpha) \geq \frac{\mathbf{1}}{\mathbf{2}}\right)^{\prime} \vee\right.$ $\mathcal{T}(v(\beta)=\mathbf{1})$.

Inference rules can be checked to hold in the translation into MELCC. 


\section{Translation of Sette logic}

Sette [18] introduced a paraconsistent logic with the aim to give a logic for "inconsistent (but not absolutely inconsistent) formal systems". Its connectives are on Table 4. In this logic, the intuitionistic nega-

\begin{tabular}{|c|c|c|c|c|c|c|c|}
\hline & $\rightarrow_{S e}$ & 0 & $\frac{1}{2}$ & 1 & $\mathrm{x}$ & $-x$ & \\
\hline & 0 & 1 & 1 & 1 & 0 & 1 & \\
\hline & $\frac{1}{2}$ & 0 & 1 & 1 & $\frac{1}{2}$ & 1 & \\
\hline & 1 & 0 & 1 & 1 & 1 & 0 & \\
\hline$\wedge_{S e}$ & 0 & $\frac{1}{2}$ & 1 & $\vee_{S e}$ & 0 & $\frac{1}{2}$ & 1 \\
\hline 0 & 0 & 0 & 0 & 0 & 0 & 1 & 1 \\
\hline$\frac{1}{2}$ & 0 & 1 & 1 & $\frac{1}{2}$ & 1 & 1 & 1 \\
\hline 1 & 0 & 1 & 1 & 1 & 1 & 1 & 1 \\
\hline
\end{tabular}

Table 4: Sette logic connectives

tion is definable as $\sim a:=a \rightarrow_{S e} 0$. The peculiarity of this logic is that conjunction and disjunction can be expressed from implication and negation as follows (they are not min and $\max$ ):

$$
\begin{aligned}
x \wedge_{S e} y & :=\left(\left(\left(x \rightarrow_{S e} x\right) \rightarrow_{S e} x\right) \rightarrow_{S e}\right. \\
& \left.-\left(\left(y \rightarrow_{S e} y\right) \rightarrow_{S e} y\right)\right) \rightarrow_{S e}-\left(x \rightarrow_{S e}-y\right) \\
x \vee_{S e} y & :=\left(x \rightarrow_{S e}--x\right) \rightarrow_{S e}\left(-x \rightarrow_{S e} y\right)
\end{aligned}
$$

The meaning of the third value is not discussed, but the connectives (see Table 4), except for negation, suggest no difference between $\mathbf{1}$ and $\frac{\mathbf{1}}{2}$. The translation of Sette connectives in MELCC is thus the same in the two cases $\geq \frac{1}{2}$ and $=\mathbf{1}$ :

$$
\begin{gathered}
\mathcal{T}\left(v\left(\alpha \rightarrow \rightarrow_{S e} \beta\right) \geq \frac{\mathbf{1}}{\mathbf{2}}\right)=\mathcal{T}\left(v ( \alpha \geq \frac { \mathbf { 1 } } { \mathbf { 2 } } ) \rightarrow \mathcal { T } \left(v\left(\beta \geq \frac{\mathbf{1}}{\mathbf{2}}\right)\right.\right. \\
\mathcal{T}\left(v\left(\alpha \wedge_{S e} \beta\right) \geq \frac{\mathbf{1}}{\mathbf{2}}\right)=\mathcal{T}\left(v ( \alpha \geq \frac { \mathbf { 1 } } { \mathbf { 2 } } ) \wedge \mathcal { T } \left(v\left(\beta \geq \frac{\mathbf{1}}{\mathbf{2}}\right)\right.\right. \\
\mathcal{T}\left(v\left(\alpha \vee_{S e} \beta\right) \geq \frac{\mathbf{1}}{\mathbf{2}}\right)=\mathcal{T}\left(v ( \alpha \geq \frac { 1 } { \mathbf { 2 } } ) \vee \mathcal { T } \left(v\left(\beta \geq \frac{1}{\mathbf{2}}\right)\right.\right.
\end{gathered}
$$

For atoms we have $\mathcal{T}\left(v(-a) \geq \frac{\mathbf{1}}{\mathbf{2}}\right)=\square a^{\prime}$ and $\mathcal{T}\left(v\left(a \rightarrow_{S e} b\right) \geq \frac{\mathbf{1}}{\mathbf{2}}\right)=\square a \rightarrow \square b ; \stackrel{\mathcal{T}}{ }\left(v\left(a \wedge_{S e} b\right) \geq\right.$ $\left.\frac{\mathbf{1}}{\mathbf{2}}\right)=\square a \wedge \square b ; \mathcal{T}\left(v\left(a \vee_{S e} b\right) \geq \frac{\mathbf{1}}{\mathbf{2}}\right)=\square a \vee \square b$. Note that this is the same translation as conjunctions and disjunctions of Priest logic (the min and the max) and Jaśkowksi implication.

An axiom system together with modus ponens is given such that the logic (named $P_{1}$ ) is complete and "cannot be strengthened (i.e., there is no propositional calculus between $P_{1}$ and $P_{0}$, where $P_{0}$ is the classical propositional calculus)". In other words, if we add to $P_{1}$ any tautology which holds in $P_{0}$ but not in $P_{1}$ we get $P_{0}$. The axioms are:
$(\mathrm{S} 1) \alpha \rightarrow_{S e}\left(\beta \rightarrow_{S e} \alpha\right)$
$(\mathrm{S} 2)\left(\alpha \rightarrow_{S e}\left(\beta \rightarrow_{S e} \gamma\right)\right) \rightarrow_{S e}\left(\left(\alpha \rightarrow_{S e} \beta\right) \rightarrow_{S e}\right.$ $\left.\left(\alpha \rightarrow_{S e} \gamma\right)\right)$
$(\mathrm{S} 3)\left(-\alpha \rightarrow_{S e}-\beta\right) \rightarrow_{S e}\left(\left(-\alpha \rightarrow_{S e}--\beta\right) \rightarrow_{S e} \alpha\right)$
(S4) $-\left(\alpha \rightarrow_{S e}-\alpha\right) \rightarrow_{S e} \alpha$
(S5) $\left(\alpha \rightarrow_{S e} \beta\right) \rightarrow_{S e}--\left(\alpha \rightarrow_{S e} \beta\right)$

and the rule of inference is modus ponens. We note that modus ponens holds also in the translation. Indeed, since we consider both $\mathbf{1}$ and $\frac{\mathbf{1}}{\mathbf{2}}$ as designated values, it corresponds to: "from $\mathcal{T}\left(v(\alpha) \geq \frac{1}{2}\right)$ and $\mathcal{T}\left(v(\alpha) \geq \frac{\mathbf{1}}{\mathbf{2}}\right) \rightarrow \mathcal{T}\left(v(\beta) \geq \frac{\mathbf{1}}{\mathbf{2}}\right)$ it follows $\mathcal{T}\left(v(\beta) \geq \frac{\mathbf{1}}{\mathbf{2}}\right)$ ", which clearly holds in MELCC.

Proposition 7.1. If $A$ is an axiom in Sette logic, then $\mathcal{T}\left(v(A) \geq \frac{\mathbf{1}}{\mathbf{2}}\right)$ is a tautology in $M E L C C$.

Proof. Lemma 1 applies to Sette Logic. Axioms (S1) and (S2) are Boolean axioms, thus they easily follow. For axiom (S3), we use recursive translation rules and come down to a formula of the form $\mathcal{T}\left(v(\alpha) \leq \frac{\mathbf{1}}{\mathbf{2}}\right) \vee \mathcal{T}\left(v(\alpha) \geq \frac{\mathbf{1}}{\mathbf{2}}\right)$, which is a tautology by lemma 1. Axioms (S4, S5) are proved in a similar manner.

The fragment of MELCC capturing Sette logic is thus: $\square a\left|\square a^{\prime}\right| \psi^{\prime}|\psi \wedge \phi| \psi \vee \phi$, the same as for $J_{3}$ and Sobociński's logics.

Sette logic connectives are definable in Eukasiewicz three-valued logic as follows [11]: $\quad \alpha \wedge_{S e} \beta:=\neg(\alpha \wedge \beta) \rightarrow_{L}(\alpha \wedge \beta)$ and $\alpha \rightarrow_{S e} \beta:=J_{0}(\beta) \rightarrow_{L} \quad J_{0}(\alpha)$, where $J_{0}(\alpha):=\neg \alpha \wedge_{S e} \neg\left(\alpha \wedge_{S e} \neg \alpha\right)$ and $\wedge$ denotes the minimum; $J_{0}$ returns 1 if and only if $\alpha=0$. The converse does not hold since with Sette connectives is not possible to obtain the value $\frac{1}{2}$.

So, even if the three 3 -valued logics $J_{3}, R M 3$ and Sette are not equivalent, they share the same paraconsistent behaviour: they can be expressed in the same MELCC fragment where the only language restriction is the presence of modalities only in front of literals, and conjunction, disjunction and implication have the same translation.

\section{The symmetry between paraconsistent and incomplete information logics}

Note that Proposition 1 says that the necessity modality $\square$ behaves like a possibility modality $\diamond$ in the sense of usual regular modal logics. Namely, exchanging the notation $\square$ and $\diamond$, the modal axioms of MELCC become

$$
\begin{aligned}
& \text { 1. } \phi \rightarrow(\psi \rightarrow \phi) \\
& \text { 2. }(\psi \rightarrow(\phi \rightarrow \mu)) \rightarrow((\psi \rightarrow \phi) \rightarrow(\psi \rightarrow \mu)) \\
& \text { 3. }\left(\phi^{\prime} \rightarrow \psi^{\prime}\right) \rightarrow(\psi \rightarrow \phi)
\end{aligned}
$$

(RE) $\square \alpha \equiv \square \beta$ if and only if $\vdash \alpha \equiv \beta$

$(\mathrm{RM}) \diamond \alpha \rightarrow \diamond \beta$ if $\vdash \alpha \rightarrow \beta$ in $\mathrm{PL}$

$(3-\mathrm{D}) \diamond \alpha \wedge \diamond \beta \wedge \diamond \gamma \rightarrow \diamond(\alpha \wedge \beta) \vee \diamond(\alpha \wedge \gamma) \vee \diamond(\beta \wedge \gamma)$

(N) $\square T$;

$(\mathrm{POS}) \diamond \top$

$(\Pi \vee) \diamond(\alpha \vee \beta) \rightarrow(\diamond \alpha \vee \diamond \beta)$

(D) $\square \alpha \rightarrow \diamond \alpha$ 
Unsurprisingly, these axioms (but for (3-D)) are typical of the KD systems, and (POS) is redundant, this time. These axioms actually characterize the fragment of KD, called MEL (Meta- or Minimal Epistemic Logic) [5]. In fact, the syntax of MELCC is exactly the same as the one of MEL. The MEL system (with Modus Ponens rule) has been proved sound and complete with respect to the following satisfiability relation, where epistemic states (nonempty sets $E \subseteq \Omega$ ) and semantic inference of a formula from a modal base $B$ :

- $E \models \square \alpha$ if $E \subseteq[\alpha]$;

- $E \models \phi \wedge \psi$ if $E \models \phi$ and $E \models \psi$;

- $E \models \phi^{\prime}$ if $E \models \phi$ is false.

- $B \models \phi$ if and only if $\forall E \subseteq \Omega$, if $E \models \psi, \forall \psi \in$ $B, E \models \phi$.

MEL is also a syntactic rendering of Boolean possibility theory. Namely, an epistemic state $E$ induces two Boolean set functions $N$ and $\Pi$ on $2^{\Omega}$ defined by $N(A)=1$ if $E \subseteq A$ and 0 otherwise (this is the necessity measure expressing the $\square$ modality here) and $\Pi(A)=1$ if $E \cap A \neq \emptyset$ and 0 otherwise (this is the possibility measure expressing the $\diamond$ modality)

We thus consider a fragment of MELC [13], which has been conceived for two possible incomplete contradictory sources, and strengthen it in order to capture completeness of information. It is easy to check that axiom (3-D) induced from (3-C) enforces epistemic states satisfying the MEL axioms to contain only one or two classical interpretations, i.e., be of the form $E=\left\{w_{1}, w_{2}\right\}$. Indeed if $E=$ $\left\{w_{1}, w_{2}, w_{3}\right\}$ then we may have $w_{1} \in A \cap B^{c} \cap C^{c}$, $w_{2} \in A^{c} \cap B \cap C^{c}, w_{3} \in A^{c} \cap B^{c} \cap C$, so that $\Pi(A)=\Pi(B)=\Pi(C)=1$ but $\Pi(A \cap B)=$ $\Pi(A \cap C)=\Pi(B \cap C)=0$.

However, with only two elements $w_{1}, w_{2}, \Pi(A)=$ $\Pi(B)=\Pi(C)=1$ implies that at least one of $\Pi(A \cap$ $B), \Pi(A \cap C), \Pi(B \cap C)$, is 1. Indeed we cannot choose $w_{1} \in A \cap B^{c} \cap C^{c}, w_{2} \in A^{c} \cap B \cap C^{c}$, since $\Pi(C)=1$, which enforces either $\Pi(A \cap C)=1$ or $\Pi(B \cap C)=1$.

The total symmetry between systems MELCC and MEL $+(3-\mathrm{D})$ can thus be viewed at the semantic level in terms of possibility theory :

\section{$\square \alpha$ holds in MELCC}

if and only if $w_{1} \in[\alpha]$ or $w_{2} \in[\alpha]$,

if and only if $\left\{w_{1}, w_{2}\right\} \cap[\alpha] \neq \emptyset$

if and only if $\diamond \alpha$ holds in MEL+(3-D).

Interestingly the MEL system is the target language for the translation of three-valued logics of incomplete information where the third truth-value means unknown (such as Kleene logic) [10]. We denote by $\mathcal{T}_{M E L}(v(a) \in T)$ the translation into MEL of the statement $v(a) \in T$, with the same understanding as in Section 3:

$$
\begin{aligned}
& \mathcal{T}_{M E L}(v(a)=\mathbf{1})=\square a \\
& \mathcal{T}_{M E L}(v(a)=\mathbf{0})=\square a^{\prime} \\
& \mathcal{T}_{M E L}\left(v(a)=\frac{\mathbf{1}}{\mathbf{2}}\right)=\diamond a \wedge \diamond a^{\prime} \\
& \mathcal{T}_{M E L}\left(v(a) \geq \frac{\mathbf{1}}{\mathbf{2}}\right)=\diamond a \\
& \mathcal{T}_{M E L}\left(v(a) \leq \frac{\mathbf{1}}{\mathbf{2}}\right)=\nabla a^{\prime}
\end{aligned}
$$

which can be compared with the translation into MELCC : $\square$ and $\diamond$ are again exchanged.

Note that the fragment of MEL obtained from translating truth-assigned formulae in three-valued logics only uses modal atoms of the form $\square \ell$, where $\ell$ is a literal [10]. As a consequence, this fragment does not contain axiom $3-\mathrm{D}$, since the latter puts $\diamond$ in front of conjunctions of atoms, and cannot be expressed in terms of a propositional formula with atoms $\square \ell$. So the presence of axiom 3-D is immaterial for the translation from three-valued logics to MEL. The same remark can be done for MELCC with respect to axiom $2-\mathrm{C}$. This is reflected by the fact that even if the number of agents can be restricted to two, this number is immaterial in the definitions of the three truth-values.

In summary, it is immaterial to choose the translation $\mathcal{T}$ into MELCC or translation $\mathcal{T}_{M E L}$ into MEL. In other words, $\mathcal{T}_{M E L}\left(v(a) \geq \frac{\mathbf{1}}{\mathbf{2}}\right)$ in MEL behaves just as $\mathcal{T}\left(v(a) \geq \frac{\mathbf{1}}{\mathbf{2}}\right)$ in MELCC in the sense that we can map sentences in a MELCC base into sentences in a MEL base by swapping $\diamond$ and $\square$, while preserving the same deductive closures (up to the renaming of modalities). More precisely, if $K$ a set of sentences in $\mathcal{L}_{\square}$, denote by $\sigma(K)$ the set of sentences obtained by swapping the two modalities $\square$ and $\diamond$. If $B=\left\{\alpha_{i}: i=1, \ldots n\right\}$ is a set of sentences in one of the paraconsistent logics mentioned above and $\mathcal{C}$ denotes the deductive closure, we have that:

$$
\mathcal{C}_{M E L}\left(\mathcal{T}_{M E L}(B)\right)=\sigma\left(\mathcal{C}_{M E L C C}(\mathcal{T}(B))\right)
$$

where $\mathcal{T}(B)=\left\{\mathcal{T}\left(\alpha_{i}\right): i=1, \ldots n\right\}$. So both MELCC and MEL + (3-D) can be used as the target language for three-valued paraconsistent logics. For instance, the fact that Sette logic is reputed to be a maximal logic less than propositional logic is retrospectively not mysterious, and can be seen in the modal translation at the semantic level. One can claim that MEL + (3-D) is really an epistemic logic expressing knowledge as little incomplete as possible, since epistemic states that serve as models contain not more than two interpretations. Enforcing an additional constraint will reduce such epistemic states to standard interpretations and leads a collapse into propositional logic.

Note that the Belnap [6] truth-values unknown and contradictory, indeed play symmetric roles in the bilattice structure (the two three-valued logics obtained by deleting one of these values coincide with a Kleene structure). 


\section{Conclusion}

This paper suggests that the two-completelyinformed-agent modal logic MELCC is a natural framework for reasoning under contradictory information in the style of several existing three-valued paraconsistent logics, when the third truth value means both true and false. The restriction to the fragment of MELCC putting modalities only in front of literals is the price paid by the truthfunctionality of these logics. MELCC thus offers an encompassing framework, where it is possible to express the fact that a conjunction is not paraconsistent while each of its conjoints is (typically the case where $w_{1} \models a \wedge b^{\prime}$ and $\left.w_{2} \models a^{\prime} \wedge b\right)$.

As MELCC may look like a baroque modal system (since the roles of the usual modalities are exchanged), it is important to point out that the modal framework for the translation is equivalently the one of MEL [5], that is, logic KD without modality nesting and purely modal formulas, provided that $\square$ and $\diamond$ are swapped in the translation. It highlights the fact that three-valued paraconsistent logics are symmetric to logics of incomplete information (for instance Priest and Kleene logics can be paired). So, the choice of the meaning of the third truth-value (unknown vs. contradictory) does not affect the translation into MEL, but for the choice between $\square$ (if $1 / 2=$ unknown) and $\diamond$ (if $1 / 2=$ contradictory) when prefixing the translations of atoms of the concerned three-valued language. It seems that given a 3 -valued logic, the choice between the two meanings of the third truth-value (unknown and contradictory) is a matter of choosing the designated truth values ( $\mathbf{1}$ for unknown and $\mathbf{1}, \frac{\mathbf{1}}{\mathbf{2}}$ for contradictory); it remains to be understood what makes one system of paraconsistent logic more attractive than another one, and what the translations into modal logic, where the meaning of implications is clear, can contribute to this issue.

Clearly, we can deal with both flaws in information, incomplete and contradictory knowledge, together. In this case, we need four values, in the style of Dunn-Belnap logic, and the next step is to formally show that the logic MELC introduced in [13] plays for Belnap logic the same role as MEL plays for Kleene logic and MELCC for Priest logic.

Acknowledgment The authors wish to thank Philippe Besnard for remarks on the first draft that led us to improve the presentation.

\section{References}

[1] A R Anderson and N D Belnap, Entailment. The logic of relevance and necessity, Princeton University Press, Princeton, New Jersey, 1975.

[2] F G Asenjo, A calculus of antinomies, Notre Dame J. Formal Log. 7 (1966), 103-105.
[3] F G Asenjo and J Tamburino, Logic of antinomies, Notre Dame J. Formal Log. 16 (1975), 17-44.

[4] A Avron, On An Implication Connective of $R M$, Notre Dame J. Formal Log. 27 (1986), 201-209.

[5] M Banerjee and D Dubois, A simple modal logic for reasoning about revealed beliefs, Proc. ECSQARU 2009, Verona, Italy, LNAI 5590 (C Sossai and G Chemello, eds.), SpringerVerlag, 2009, pp. 805-816.

[6] N D Belnap, A useful four-valued logic, Modern Uses of Multiple-Valued Logic (J M Dunn and G Epstein, eds.), D. Reidel, 1977, pp. 8-37.

[7] R Brady, Completeness proofs for the systems RM3 and BN4, Logique et Analyse 25 (1982), 51-61.

[8] B. F. Chellas, Modal logic: an introduction, Cambridge University Press, 1980.

[9] D Ciucci and D Dubois, Relationships between connectives in three-valued logics, Proc. IPMU Conference, Catania, CCIS, vol. 297, Springer, 2012, pp. 633-642.

[10] , Three-valued logics for incomplete information and epistemic logic, Proc. 13th European Conference on Logics in Artificial Intelligence (JELIA), Toulouse, France, LNCS, vol. 7519, 2012, pp. 147-159.

[11] N C A da Costa and E H Alves, Relations between paraconsistent logics and many-valued logic, Bull. Sec. Logic Polish Acad. Sci. 10 (1981), 185-191.

[12] I M L D'Ottaviano and N C A da Costa, Sur un problème de Jaśkowski, Comptes Rendus de l'Académie des Science 270 (1970), 1349-1353.

[13] D Dubois, Reasoning about ignorance and contradiction: many-valued logics versus epistemic logic, Soft Comput. 16 (2012), no. 11, $1817-$ 1831.

[14] S Jaśkowski, Propositional calculus for contradictory deductive systems, Studia Logica $\mathbf{2 4}$ (1969), 143-160.

[15] J Lawry and I González Rodríguez, A bipolar model of assertability and belief, Int. J. Approx. Reasoning 52 (2011), no. 1, 76-91.

[16] W. Carnielli M. Lima-Marques, Society semantics for multiple-valued logics, Advances in Contemporary Logic and Computer Science, Contemporary Mathematics, vol. 235, American Mathematical Society, 1999, pp. 33-52.

[17] G Priest, The Logic of Paradox, J. Philos. Logic 8 (1979), 219-241.

[18] A M Sette, On propositional calculus $P_{-}$1, Math. Japon. 16 (1973), 173-180.

[19] B Sobociński, Axiomatization of a partial system of three-value calculus of propositions, J. of Computing Systems 1 (1952), 23-55.

[20] K. Tanaka, F. Berto, E. Mares, and F. Paoli (eds.), Paraconsistency: Logic and applications, Springer, 2013. 